\title{
Experimenting with technology associated with Mobile Desktops
}

\author{
Maria H. Penedo
}

\begin{abstract}
.
Tomorrow's ways of doing business are likely to be far more challenging and interesting than today's due to technological advances that allow people to operate or cooperate anytime, anywhere. Today's workers are becoming mobile without the need of a work home base. Organizations are evolving from the hierarchical lines of control and information flow into more dynamic and flexible structures, where "teams" and individuals are the building blocks for forming task forces and work groups to deal with short and long term project tasks, issues and opportunities. Those individuals and teams will collaborate from their mobile desktops, whether at their offices, home or on the road. A revised paradigm for conducting small and large-scale development and integration is emerging, sometimes called the "virtual enterprise", both in the military and industrial environments. This new paradigm supports communication, cooperation and collaboration of geographically dispersed teams. In this paper we discuss experiences with specific technologies that were investigated by TRW's Infrastructure for Collaboration among Distributed Teams (ICaDT) project; an Independent Research and Development (IR\&D) effort.
\end{abstract}

\section{Introduction}

TRW and its customers have been increasingly moving to evolutionary acquisitions which require collaboration among U.S. Government interests (materiel developers, combat developers, and geographically distributed agencies), multiple participating contractors and subcontractors, and distributed teams. Specific problems with distributed projects include: difficulties in communication; sharing data generated at distributed sites; finding appropriate data; collaborating in "real-time" with full access to electronic data; replicated email with no reply-connectivity describing history or progression of ideas and issues; keeping track of information flow. In order to solve or mitigate those problems, existing processes need to be re-engineered to include the appropriate technical and social aspects and be supported by the appropriate automated infra-structures to enable creation of such virtual enterprise. A central part of this paradigm is the concept of collaboration from "mobile" desktops, which may be the office, home or a hotel room, as depicted in Figure 1.

The ICaDT project was created with the goal to improve the ability of geographically dispersed teams and organizations to rapidly respond to continuous change and new opportunities by using collaborative process technologies. This paper presents and discusses results of the ICaDT project investigations which are applicable to this virtual enterprise paradigm in support of distributed development; further information can be found in [PeHe98]. In the past, we have experimented with process automation support for projects in a non-distributed environment [Pe94, Pe94a, Pe93, PeSh91] .

\section{The ICaDT Project}

The Infrastructure for Collaboration among Distributed Teams (ICaDT) project has varied from having distributed and co-located players. It has created a "virtual group", the extended team consisting of distributed players residing in different locations in California, Kentucky and Oklahoma. We are using our own technology for collaborating within the core team on our daily activities and we often use it with the extended team; its usage has positively impacted our internal way of doing business, especially with respect to agility and productivity improvement. 
The overall objective of the ICaDT project is to improve the ability of geographically dispersed teams and organizations to: a) rapidly respond to continuous change and opportunities, and b) to function more effectively, via supporting technologies. Key goals are: to increase productivity and save time by having rapid access to people and resources; to decrease costs, by avoiding travel; and to make people more efficient, by providing them with automation support and availability of resources from their desktops. In other words, support the needs of anytime, anywhere collaboration, as depicted in Figure 1. Key objectives include to:

- Experiment with key ingredients/elements of the "on-line collaboratory" (a virtual project library of process, data, and tools) of the future.

- Prototype new capabilities for visualizing the management, structure, and behavior of intrinsically distributed systems

- Seed new projects with collaboration infrastructure prototype technology, and harvest the improvements they make and the lessons they learn into enhanced TRW processes.

The initial focus of the project was driven by the needs of TRW personnel and concentrated on areas generally applicable to the full life-cycle process and team collaboration. The value-added areas selected for experimentation/validation are: synchronous desktop conferencing; asynchronous collaboration, integration of synchronous and asynchronous capabilities into virtual collaborative spaces or virtual rooms; and workflow as it relates to collaboration. The desktop conferencing technology has been assessed and validated and recommendations have been made; the Virtual Room technology is in its second phase of experimentation. Workflow capabilities in support of managed team collaboration is currently being explored.

An initial vision of a collaborative, distributed enterprise organization for large, multi-agency, multicontractor, geographically dispersed programs has been created. The vision characterizes the evolution of group interaction from the present, through the incorporation of collaborative technologies, to an anticipated future in which on-line collaboratories are commonplace, and shared virtual spaces are the norm for computer-assisted collaboration. In the future vision, depicted in Figure 2, the enterprise will support rapid formation and re-formation of possibly distributed teams, each with focused objectives, organized into dynamic hierarchies (teams of teams), relying on innovative management discipline for coordination. People will have immediate access to data and people independent of their location. Data and applications can be organized by subject or team objectives and available in virtual spaces where people can visit at any time and either chat or collaborate with other team members present in those spaces at that time, or have access and/or generate the latest information pertinent to that team.

As projects or organizations change, the process and supporting tools must support this dynamism. Thus, another important ingredient in support of this "virtual enterprise" of the future is a management process architecture supported by collaboratories. This process architecture should include process characterizations and support dynamic group formation, relying on innovative management disciplines for coordination. This process architecture may depend on commonly accessible "knowledge repositories" of processes and products providing role-specific views of the distributed project information base as it evolves. The ICaDT Virtual Collaboratory, described next, can be considered an initial experimental framework for this vision; it includes recommendations on immedi- 
ately-available capabilities to support the process architecture, and "lessons learned" to guide future work.

\section{The ICaDT Collaboratory.}

The ICaDT Collaboratory is a simplified instantiation of a futuristic working space for collaboration purposes. It currently is a testbed of hardware and software products, including NT and Solaris servers, which lives in virtual space and hosts collaborative technologies for experimentation and use. It is also the ICaDT project knowledge repository, i.e., it gathers and make available all the products generated during the course of this IR\&D project. The collaboratory provides an infrastructure for anytime, anywhere access to people and data. The ICaDT Collaboratory, illustrated in Figure 3, includes:

- Two virtual conference centers, denoted ICaDT Conference Center \#1 and \#2 respectively; their technology is described in Section 5 . These conference centers have 58 ports each, thus allowing 58 concurrent users to conference with each other in one or separate conferences. These conference centers are web-based, thus can be accessed from a web browser from anywhere within the TRW intranet. With the appropriate mechanisms, they can be accessed from outside the firewall by selected and pre-defined sites. Free conferencing client plug-ins can be downloaded from those centers. Each conference center is the "neT.120" COTS product, from Databeam Corporation, which was selected as a by-product of our assessments.

- A virtual building which hosts virtual rooms to be used by teams which need to collaborate. There are currently 7 rooms for experimentation. Each virtual room in the Collaboratory corresponds to an instantiation of a web-based prototype developed by the project. The rooms are currently being used by selected projects, IPTs and working groups in support of their collaborative exchanges.

- A virtual data repository, also web-based, which includes:

- data about collaborative technology in general and pointers to appropriate vendors and documents, and

- information generated by the project including: process scenarios, concepts, instructions, lessons learned, prototype descriptions, technical evaluations.

- A toolkit for building collaborative applications. This collaborative tool-kit is a building block for building target systems which include collaborative capabilities. This is a new area for investigation.

\section{Desktop Conferencing Technical Results.}

The ICaDT project has experimented with a number of tools and approaches for desktop usage and recommends immediate use of a client-server approach to synchronous data conferencing, over IPnetworks, as illustrated in Figure 4. The server connects and moves data among the various sites, thus improving performance over multi-client connections; clients have the viewing and annotation capabilities. This approach does not preclude having one of the sites be a meeting room. Since lowcost integrated audio and video over IP do not yet provide needed quality of service, we recommend the telephone as the vehicle for audio communication. With the adoption of the new H323 standard (audio/video over IP-networks), it is expected that those capabilities will be available in the near future. Those client and server technologies are evolving quickly and hopefully will become interoper- 
able as the standards solidify. Note that the market place has a wide number of those tools as listed in various assessments, such as [CoKw95, IPW], which vary in capability and cost.

Clients recommended are T.120-compliant market leaders as conferencing tools: Microsoft's NetMeeting (PC market leader), which comes free with Microsoft Office; Databeam's Farsite or free plug-in Farsite Lite; or Intel's Proshare. Client tool interoperability is still very weak, restricted to file transfer capabilities; interoperability will come with the standardization of the various functions and the implementation of such standards.

The conference server proposed for immediate use is Databeam's neT.120. It supports the following requirements defined based on our goals of providing every TRW personnel with desktop capabilities:

- Desktop access, anytime, anywhere. NeT.120 is web-based.

- Easy to use. It takes 2-4 clicks to attend a conference in virtual space.

- Multi-user. Our servers support up to 58 concurrent users; the limit is the number of ports in server, which can be expanded via networks of servers.

- Multi-platform (PC, Mac, Unix). Compatible conferencing tools fully support PCs and partially support Mac/Unix (with forthcoming full support).

- Cost-effective per user ( $<\$ 200 /$ user). NeT.120 costs $\$ 10$ to $\$ 20$ per user or less since each port typically can support up to 10 real users.

- Robust. We experienced only one neT.120 server crash during 6 months of experimentation. The Farsite client sometimes freezes (quite typical with Windows systems) but the conference state can be easily recovered by re-joining the conference via the web browser.

- Standards compliant. NeT.120 supports T.120 (the data conferencing set of standards); H.323 forthcoming (audio/video)

- Vendor positioning. Many vendors are including the neT.120 server either as part of their product (e.g., Lucent) or are interfacing with it (e.g., Microsoft).

- Functionality. It not only provides the multi-user broadcast server functionality but it includes multi-platform viewers as well as Java application sharing capabilities.

The T120 protocol introduces issues when being used across firewalls; alternative approaches have been investigated and are proposed in [Ca97].

\section{Results of Virtual Rooms Experimentation.}

The ICaDT project has initiated experimentation with virtual spaces in the context of "virtual rooms for small interest teams". This virtual room is a meeting place in virtual space where teams can exchange information as depicted in Figure 5 . It is like a proposal room in virtual space (thus accessible from a desktop - anytime, anywhere) for permitted members, where data is always available there (news, latest decisions, documents, actions) and in addition maintains threads of asynchronous discussions; people come and go in that virtual space and can chat/collaborate with other people present or modify data an leave it available to others.

Single-functionality chat rooms are in wide use on the Web today. More advanced virtual rooms are being explored by the research community and were considered either too complex or too premature for our experiments with "real" users. Examples or research systems are: Mitre's Collaborative Virtual Workspace (CVW) [Sp96], and the University of Queensland's Orbit system and the University 
of Illinois' Virtue system [KaRe97]. Virtual rooms dedicated to engineering or technical activities need capabilities appropriate to their needs. In order to experiment with the virtual room concept and based on the needs of the internal collaborative working group, a web-based prototype of a virtual room was developed, to support the following requirements: web-based, COTS-based, easiness of instantiation, easiness of use, immediate availability for experimentation, and almost free.

The ICaDT VR prototype's provides the following functional capabilities: I) names of people "virtually" present in room; ii) bulletin board containing news including announcements; discussion thread repository; virtual conference server for synchronous communication including chat to all and whisper to one, shared whiteboard and application sharing; shared team data repository including action, meeting minutes, files/documents, and links. Another objective of the prototype was to explore architectural and integration issues. This prototype integrates the WebBoard product , (for chat and discussion threads), HTML (for user interface and form entry) and Perl scripts (for maintaining a data repository of data actions, meeting minutes, file and urls). WebBoard 2.0 is used as the foundation for the virtual room prototype. This product was chosen based on its features, ease of use and low price $(<\$ 500)$. This prototype is in the early stages of validation. Seven rooms were established and are undergoing experimentation; they are being used for testing (room \#1), and by project teams (ICaDT, FBCB2 Top Team, CAETI), and IPTs (ATO, MSO Top Team, TechnoNet). A lobby (or directory of rooms) exists to simplify navigation among rooms. While most people are likely to be members of only one room, nothing precludes membership in multiple rooms.

We gathered lessons learned which are being published in the Knowledge Repository of the ICaDT Collaboratory; some of those lessons are:

- Extensibility and tailorability are a must in COTS applications since it is unlikely that all required features are built-in.

- Scalability must be addressed at the start to avoid complications later.

- Resource allocation (bandwidth and storage space) needs to be addressed, predicted and provided for to support growth.

- Standardizing as many features as possible across all "rooms" minimizes maintenance.

- Persistent storage issues must be addressed such as data expiration, backup and retrieval.

- Virtual rooms should be able to support a full complement of security mechanisms (e.g. encryption, authentication)

- Platform/OS independence is necessary especially with TRW's 50/50 split of PC and Mac with some UNIX clients too. HTTP (Web) and NNTP (Newsgroup discussion threads) provide that.

- Web browsers exhibit varying behavior across different platforms and version numbers. One should design for the minimum expected browser capabilities to allow wide-scale usage.

- New technologies appear everyday: audio/video streaming, push technology, agents, etc. A virtual room should be extensible enough to add yet to be determined features.

- Perl language appears to be the most mature and well suited for CGI server-side programming.

\section{Conclusions.}

This paper described activities and results of the ICaDT IR\&D in the area of Collaborative Technology. Our evaluations determined that many capabilities required of the vision and process architecture cannot be supported by today's COTS products, but that it is wise, in areas like data conferencing, that the company starts using current incomplete products with plans to evolve as the technology matures. Other areas, like virtual rooms, require changes in behavior and operation and should 
be used experimentally so we can more fully understand the impact of this technology. The market place also seems to be moving in that direction and we predict that products will be available in a year time frame.

The project has successfully carried desktop conferencing technology through assessment, experimentation and initial adoption and is on a second phase of assessment of virtual rooms. Challenges to widespread usage include: having the appropriate infra-structure (e.g., platform, net access, software version); keeping up with rapidly evolving technology; people's unwillingness to change their habits and people's unwillingness to share information ("owning information means power"). Some mitigation approaches identified in support of widespread dissemination are as follows: a) start with technology available now; b) identify incremental/evolution plans; c) coordinate with company infrastructure groups; d) identify champions to spearhead the usage; e) provide hands-on training using the technology itself; f) facilitate getting necessary tools/infrastructure.

Other few remarks with respect to technology adoption are as follows:

- Technology does not solve mundane and social problems like: time management, people management, tardiness in passing out documents for review. If anything, technology may make us improve related processes (e.g., templates for related presentations, charts prepared ahead of time)

- The learning curve for this technology is short but frequency of usage is key.

- People processes do get modified; and improved; but users need to make that first transition step.

We are currently working with another small distributed development project in order to identify further needs of such projects. 


\section{Bibliography}

- [PeHe98] Penedo, Maria H. and N. Heske, Collaborating from Mobile Desktops, TRW Technical Review Journal, to appear July 1998.

- [Pe94] Penedo, Maria H., Life-cycle (Sub) Process Scenario for 9th International Software Process Workshop (ISPW9), Proceedings of the 9th International Software Process Workshop, IEEE Computer Society Press, Arlie, Virginia, USA, Oct 1994.

- [Pe94a] Penedo, M.H., Lessons Learned in Designing and Implementing Life-Cycle Generic Models", Proceedings of the Database and Software Engineering Workshop, Sorrento, Italy, May 1994.

- [Pe 93] Penedo, M.H., and N. Madhavji, Evolution of Software Processes:Guest Editors, Special Section, IEEE Transactions on Software Engineering, Vol. 19, No. 12, December 1993.

- [PeSh91] Penedo, M.H., C. Shu, Acquiring Experiences with the Modeling and Implementation of the Project Life-cycle Process - the PMDB work, IEE Software Engineering Journal, September 1991.

- [Ca97] Campbell, Douglas, Securing T.120 Conference Servers, Collaborative Web Site, http://icadt.dh.trw.com/icadt, December 1997.

- [CoKh95\} Coleman, David and Raman Khanna, Groupware: Technology and Applications, Published by Prentice Hall, June, 1995, ISBN 0-13-305194-3. Also chapter in http://www.collaborate.com/publications/chapt_toc.html

- [KaRe97] Kaplan, Simon and D. Reed, Collaboration Support for the Development and Evolution of Complex Systems, ACM SIGSOFT Software Engineering Notes, Vol. 22, no. 5, September 1997

- [Sp96] Peter Spellman, Collaborative Virtual Workspace (CVW), Demonstration Presented at CSCW'96, http://www.mitre.org/resources/centers/advanced_info/g04e/cvw.html

- $[\mathrm{IPW}]$ Internet Product Watch: List of conferencing products: http://ipw.internet.com/conferencing/index.html 
Figures for Penedo's paper

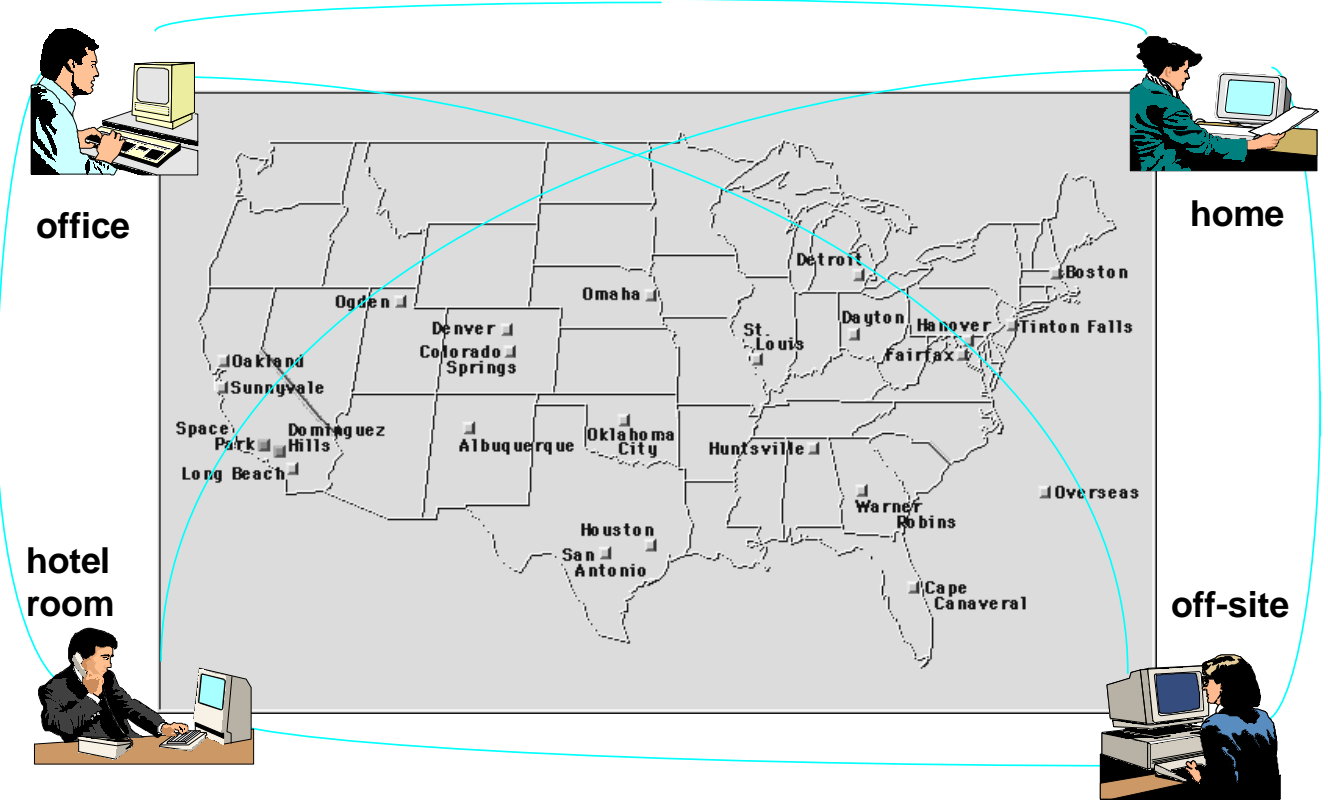

Figure 1. Anytime, anywhere collaboration

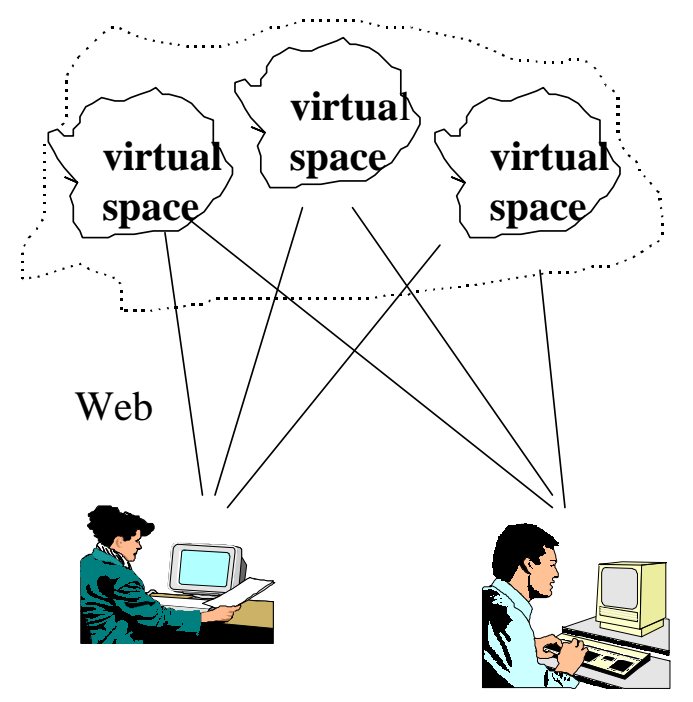

Figure 2. Processes of the Future 


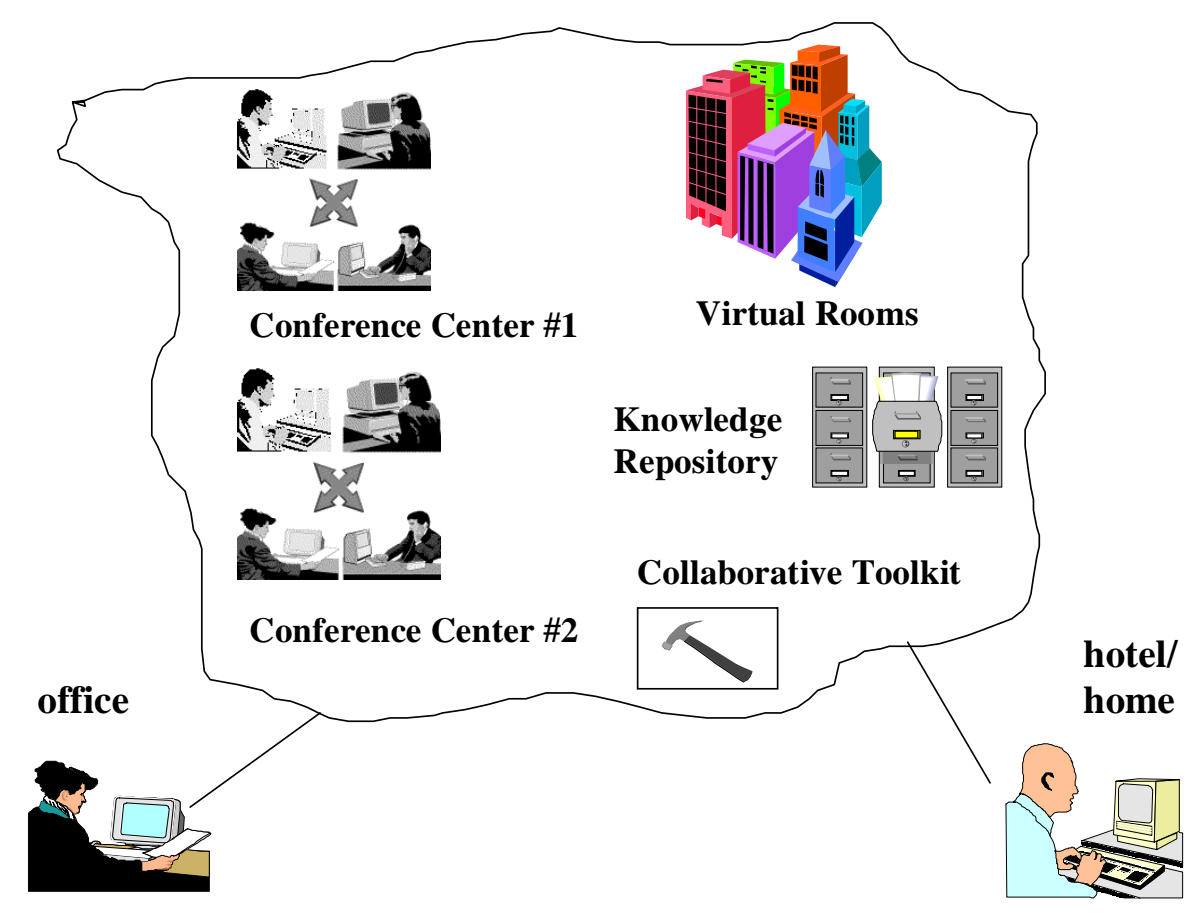

Figure 3. The ICaDT Virtual Collaboratory

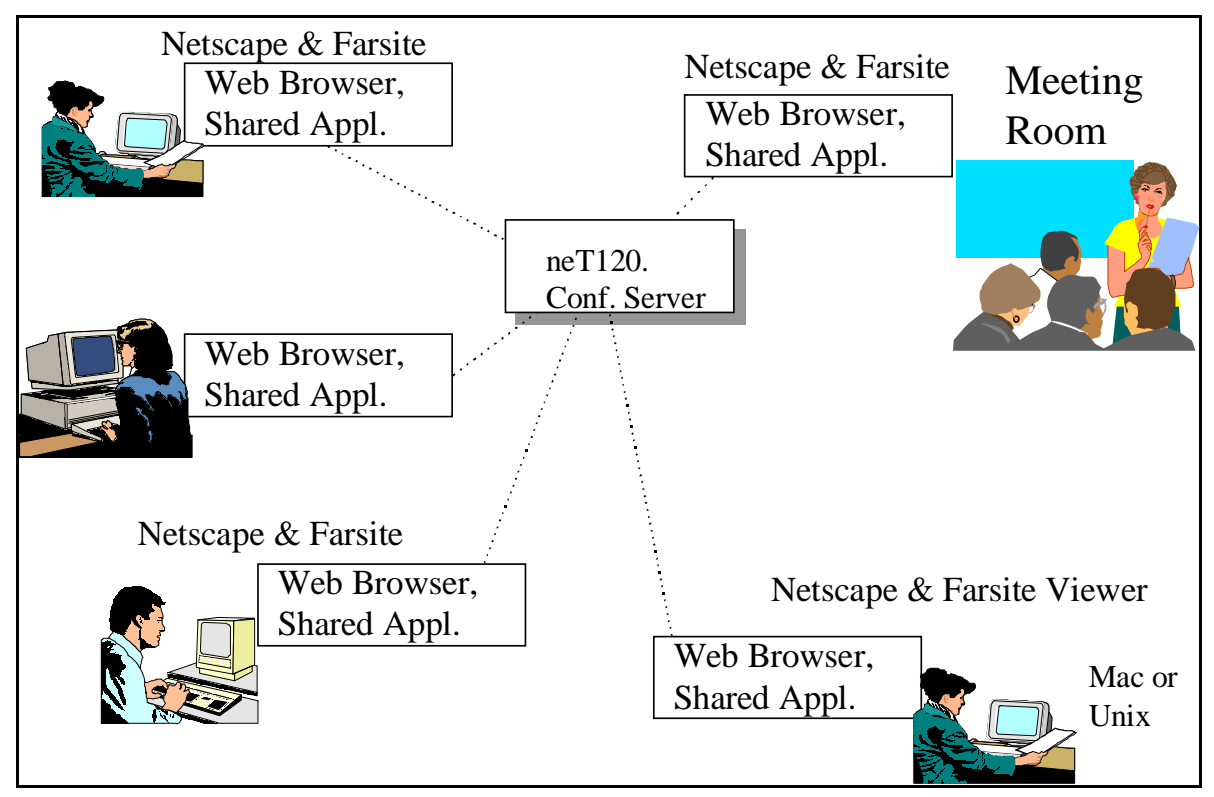

Figure 4. Conference Server Architecture 


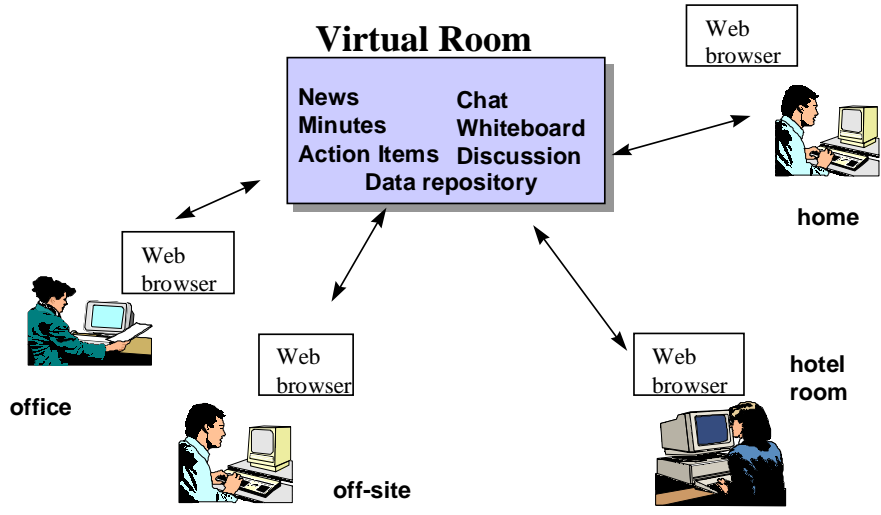

Figure 5. Web-based Virtual Room for Small Teams 